\title{
Analysis of Weighted Multifrequency MUSIC-Type Algorithm for Imaging of Arc-Like, Perfectly Conducting Cracks
}

\author{
Young-Deuk Joh \\ Department of Mathematics, Kookmin University, Seoul 136-702, Republic of Korea \\ Correspondence should be addressed to Young-Deuk Joh; mathea421@kookmin.ac.kr
}

Received 20 June 2013; Revised 24 September 2013; Accepted 2 October 2013

Academic Editor: Suh-Yuh Yang

Copyright ( 2013 Young-Deuk Joh. This is an open access article distributed under the Creative Commons Attribution License, which permits unrestricted use, distribution, and reproduction in any medium, provided the original work is properly cited.

\begin{abstract}
The main purpose of this paper is to investigate the structure of the weighted multifrequency multiple signal classification (MUSIC) type imaging function in order to improve the traditional MUSIC-type imaging. For this purpose, we devise a weighted multifrequency MUSIC-type imaging function and examine a relationship between weighted multifrequency MUSICtype function and Bessel functions of integer order of the first kind. Some numerical results are demonstrated to support the survey.
\end{abstract}

\section{Introduction}

Inverse problem, which deals with the reconstruction of cracks or thin inclusions in homogeneous material (or space) with physical features different from space, is of interest in a wide range of fields such as physics, engineering, and image medical science which are closely related to human life; refer to [1-9]. That is why inverse problem has been established as one interesting research field. Compared to the early studies on inverse problem in which much research had been done theoretically, in recent studies, more practical and applicable approaches have been undertaken and the reconstructive way appropriate to each specific study field started to be investigated thanks to the development of computational science using not only computers but also mathematical theory. As we can see through a series of papers $[10,11]$, the reconstruction algorithm, based on the iterative scheme such as Newton's method, has been mainly studied. Generally, in regard to algorithms using Newton's method, in the case of the initial shape quite different from the unknown target, the reconstruction of material leads to failure with the nonconvergence or yields faulty shapes even after the iterative methods are conducted. Hence, in such an iterative method, several noniterative algorithms have been proposed as a way to find the shape of initial value close to that of the unknown target as quickly as possible.

The noniterative algorithms such as multiple signal classification (MUSIC), subspace migrations, topological derivative, and linear sampling method can contribute to yielding the appropriate image as an initial guess. Previous attempts to investigate MUSIC-type algorithm presented various experiments with the use of MUSIC-type algorithm. For instance, the use of MUSIC-type algorithm for eddy-current nondestructive evaluation of three-dimensional defects [12], and MUSIC-type algorithm designed for extended target, the boundary curves which have a five-leaf shape or big circle was presented [13]. In addition, MUSIC-type algorithm was introduced for locating small inclusions buried in a half space [2] and for detecting internal corrosion located in pipes [3]. Although the past phenomena about experimental results could not be theoretically explained because the mathematical structure about these algorithms was not verified, recent studies [14-19] managed to partially analyze the structure of some algorithms. On the basis of these studies, the present study examined the structure of algorithms to make improvements in imaging the defects. Therefore, this paper aims to improve traditional MUSIC-type imaging algorithm by weighting applied to each frequencies.

This paper is organized as follows. In Section 2, we discuss two-dimensional direct scattering problem in the presence of perfectly conducting crack and MUSIC-type algorithm. In Section 3, we introduce a weighted multifrequency MUSICtype imaging algorithm and analyze its structure to confirm that it is an improved version of traditional MUSIC algorithm. In Section 4, we present several numerical experiments 


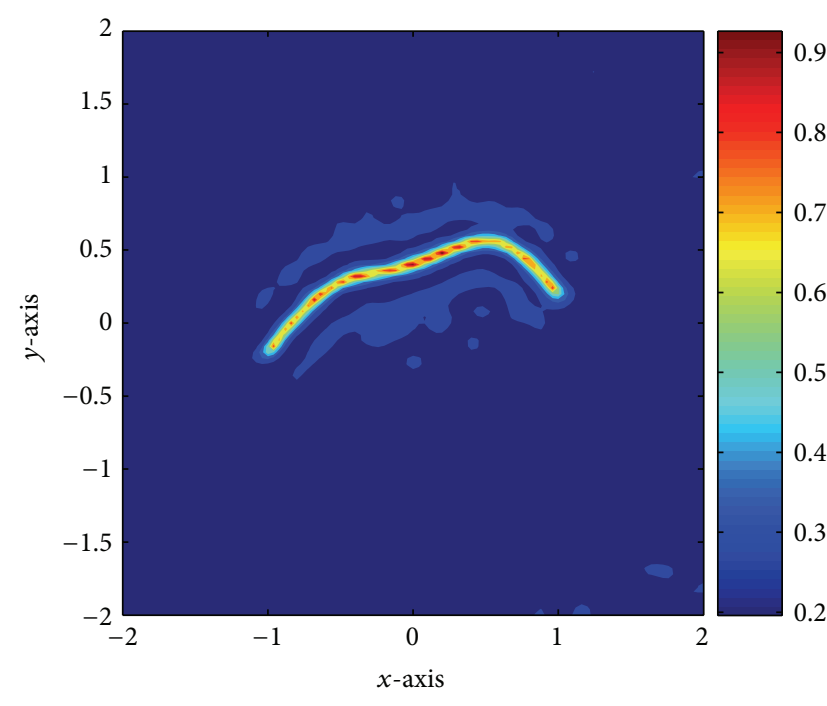

(a) Map of $\mathbb{E}_{\mathrm{MF}}(\mathbf{z} ; 10)$

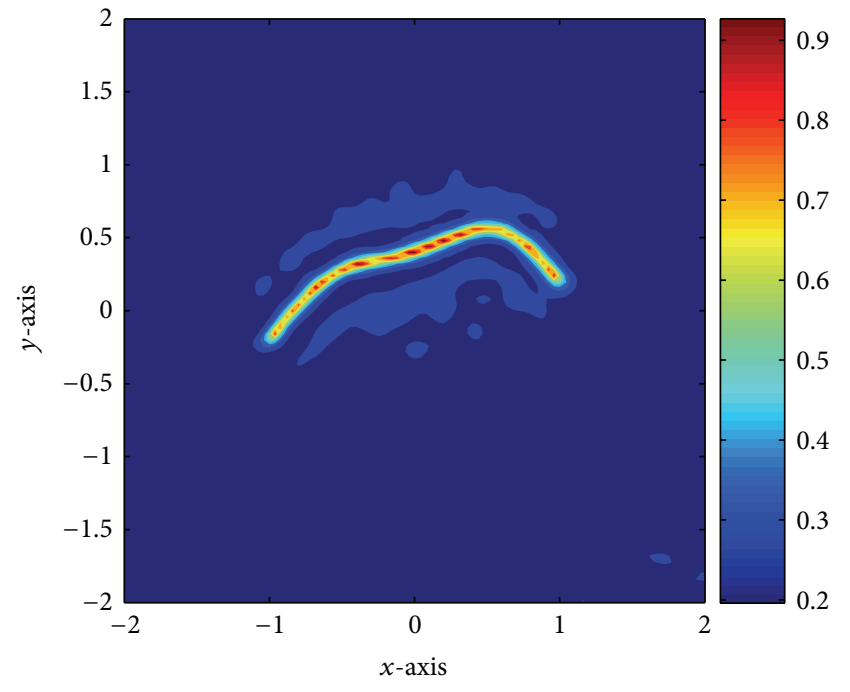

(b) Map of $\mathbb{E}_{\mathrm{WMF}}(\mathbf{z} ; 10)$

FIGURE 1: Shape reconstruction of $\Omega_{1}$ via MUSIC algorithm.

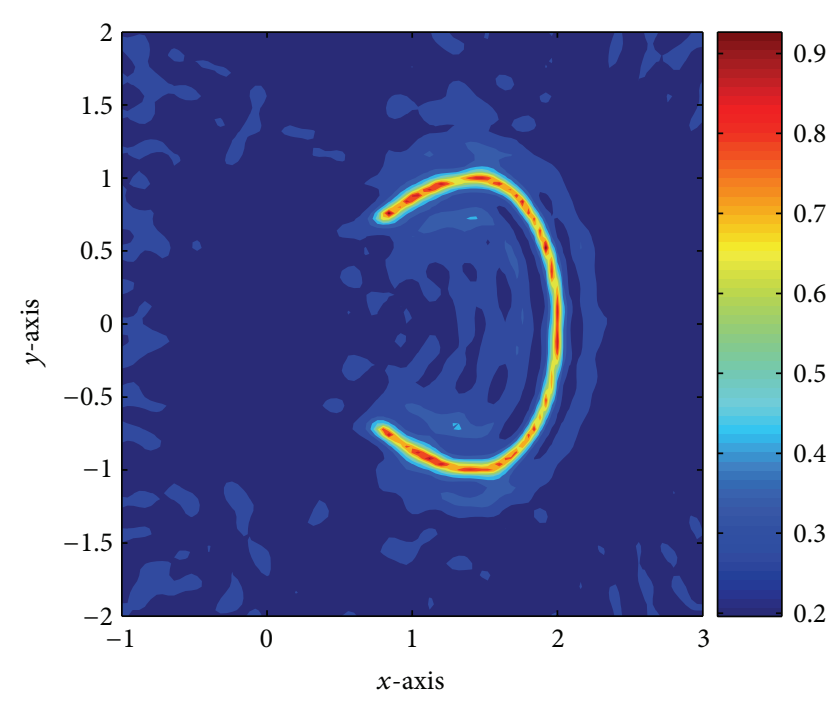

(a) Map of $\mathbb{E}_{\mathrm{MF}}(\mathbf{z} ; 10)$

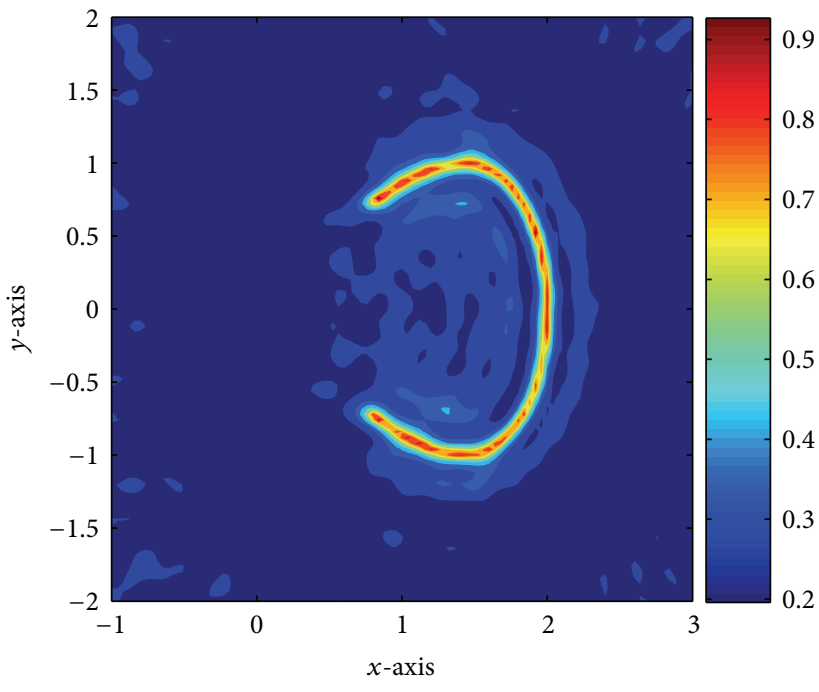

(b) Map of $\mathbb{E}_{\mathrm{WMF}}(\mathbf{z} ; 10)$

Figure 2: Same as Figure 1 except the crack is $\Omega_{2}$.

with noisy data. In Section 5, our conclusions are briefly presented.

\section{Direct Scattering Problem and Single- and Multifrequency MUSIC-Type Algorithm}

In this section, we simplify surveying the two-dimensional direct scattering problem for the existence of perfectly conducting cracks and the single- and multifrequency MUSIC algorithm. For more information, see $[10,20]$.

2.1. Direct Scattering Problem and MUSIC-Type Imaging Function. First, we consider the two-dimensional electromagnetic scattering by a perfectly conducting crack located in the homogeneous space $\mathbb{R}^{2}$. Throughout this paper, we assume that the crack $\Omega$ is a smooth, nonintersecting curve, and we represent $\Omega$ such that

$$
\Omega=\{\xi(t): t \in[-1,1]\},
$$

where $\xi:[-1,1] \rightarrow \mathbb{R}^{2}$ is an injective piecewise smooth function. We consider only the transverse magnetic (TM) polarization case. Let us denote $u_{\text {total }}$ to be the time-harmonic total field, which can be decomposed as

$$
u_{\text {total }}(\mathbf{x})=u_{\text {incident }}(\mathbf{x})+u_{\text {scatter }}(\mathbf{x}) \text {, }
$$

where $u_{\text {incident }}(\mathbf{x})=\exp (j \omega \boldsymbol{\theta} \cdot \mathbf{x})$ is the given incident field with incident direction $\boldsymbol{\theta} \in \mathbb{S}^{1}$ (unit circle) and $u_{\text {scatter }}(\mathbf{x})$ 
is the unknown scattered field that satisfies the Sommerfeld radiation condition

$$
\lim _{|\mathbf{x}| \rightarrow \infty} \sqrt{|\mathbf{x}|}\left(\frac{\partial u_{\text {scatter }}(\mathbf{x})}{\partial|\mathbf{x}|}-j \omega u_{\text {scatter }}(\mathbf{x})\right)=0
$$

uniformly in all directions $\widehat{\mathbf{x}}=\mathbf{x} /|\mathbf{x}|$. Now, the total field $u_{\text {total }}$ satisfies the two-dimensional Helmholtz equation

$$
\begin{gathered}
\Delta u_{\text {total }}(\mathbf{x})+\omega^{2} u_{\text {total }}(\mathbf{x})=0 \quad \text { in } \mathbb{R}^{2} \backslash \Omega, \\
u_{\text {total }}(\mathbf{x})=0 \text { on } \Omega
\end{gathered}
$$

with a given positive frequency $\omega$. In the case that $\Omega$ is absent, incident field $u_{\text {incident }}$ can also be a solution of (4).

The far-field pattern is defined as function $u_{\text {far }}(\widehat{\mathbf{x}}, \boldsymbol{\theta})$ that satisfies

$$
\begin{aligned}
u_{\text {scatter }}(\mathbf{x}, \omega) & =\frac{\exp \left(j k_{0}|\mathbf{x}|\right)}{\sqrt{|\mathbf{x}|}} u_{\text {far }}(\widehat{\mathbf{x}}, \boldsymbol{\theta})+o\left(\frac{1}{\sqrt{|\mathbf{x}|}}\right) \\
& =\frac{\exp (j \omega|\mathbf{x}|)}{\sqrt{|\mathbf{x}|}} u_{\text {far }}(\widehat{\mathbf{x}}, \boldsymbol{\theta})+o\left(\frac{1}{\sqrt{|\mathbf{x}|}}\right)
\end{aligned}
$$

as $|\mathbf{x}| \rightarrow \infty$ uniformly on $\widehat{\mathbf{x}}$. Then, based on [21], the farfield pattern $u_{\text {far }}(\widehat{\mathbf{x}} ; \boldsymbol{\theta})$ of the scattered field $u_{\text {scatter }}(\mathbf{x})$ can be expressed by the following equation:

$$
u_{\mathrm{far}}(\widehat{\mathbf{x}} ; \boldsymbol{\theta})=-\frac{\exp (j \pi / 4)}{\sqrt{8 \pi \omega}} \int_{\Omega} \exp (-j \omega \widehat{\mathbf{x}} \cdot \mathbf{y}) \varphi(\mathbf{y} ; \boldsymbol{\theta}) d \mathbf{y},
$$

where $\varphi(\mathbf{y} ; \boldsymbol{\theta})$ is an unknown density function (see [10]).

Second, we present the traditional MUSIC-type algorithm for imaging of perfectly conducting cracks. For the sake of simplicity, we exclude the constant $-\exp (\pi / 4) / \sqrt{8 \pi k}$ from formula (6). Based on [20, 22], we assume that the crack is divided into $M$ different segments of size of the order of half the wavelength $\lambda / 2$. Having in mind the Rayleigh resolution limit for far-field data, only one point at each segment is expected to contribute to the image space of the response matrix $\mathbb{K}$ (i.e., see $[20,22,23]$ ). Each of these points, say $\mathbf{y}_{m}, m=1,2, \ldots, M$, will be imaged via the MUSIC-type algorithm. With this assumption, we perform the following singular value decomposition (SVD) of the multistatic response (MSR) matrix $\mathbb{K}=\left[u_{\text {far }}\left(\widehat{\mathbf{x}}_{i} ; \boldsymbol{\theta}_{l}\right)\right]_{i, l=1}^{N} \in$ $\mathbb{C}^{N \times N}$ :

$$
\mathbb{K}=\mathbb{U} \mathbb{S V}_{m}^{*}=\sum_{m=1}^{M} \tau_{m} \mathbf{U}_{m} \mathbf{V}_{m}^{*}
$$

where superscript $*$ is the mark of Hermitian, $\mathbf{U}_{m}$ and $\mathbf{V}_{m} \in$ $\mathbb{C}^{N \times 1}$ are, respectively, the left- and right-singular vectors of $\mathbb{K}$, and $\tau_{m}$ denotes singular values that satisfy

$$
\tau_{1} \geq \tau_{2} \geq \cdots \geq \tau_{m}>0, \quad \tau_{m}=0, \text { for } m \geq M+1 .
$$

If so, $\left\{\mathbf{U}_{1}, \mathbf{U}_{2}, \ldots, \mathbf{U}_{M}\right\}$ are the basis for the signal and $\left\{\mathbf{U}_{M+1}, \mathbf{U}_{M+2}, \ldots, \mathbf{U}_{N}\right\}$ span the null space of $\mathbb{K}$, respectively. Therefore, one can define the projection operator onto the null subspace, $\mathbf{P}_{\text {noise }}: \mathbb{C}^{N \times 1} \rightarrow \mathbb{C}^{N \times 1}$. This projection is given explicitly by

$$
\mathbf{P}_{\text {noise }}:=\mathbb{\square}_{N}-\sum_{m=1}^{M} \mathbf{U}_{m} \mathbf{U}_{m}^{*}
$$

where $\rrbracket_{N}$ denotes the $N \times N$ identity matrix. For any point $\mathbf{z} \in \mathbb{R}^{2}$, we define a test vector $\mathbf{f}(\mathbf{z}, \omega) \in \mathbb{C}^{N \times 1}$ as

$$
\begin{aligned}
& \mathbf{f}(\mathbf{z}, \omega) \\
& \quad=\left[\exp \left(j \omega \boldsymbol{\theta}_{1} \cdot \mathbf{z}\right), \exp \left(j \omega \boldsymbol{\theta}_{2} \cdot \mathbf{z}\right), \ldots, \exp \left(j \omega \boldsymbol{\theta}_{N} \cdot \mathbf{z}\right)\right]^{T} .
\end{aligned}
$$

Based on this, we can design a MUSIC-type imaging function $W: \mathbb{C}^{N \times 1} \rightarrow \mathbb{R}$ such that

$$
\mathbb{E}(\mathbf{z})=\left\|\mathbf{P}_{\text {noise }}(\mathbf{f}(\mathbf{z}, \omega))\right\|^{-1}=\frac{1}{\left\|\mathbf{P}_{\text {noise }}(\mathbf{f}(\mathbf{z}, \omega))\right\|} .
$$

Then, the map of $\mathbb{E}(\mathbf{z})$ will have peaks of large and small magnitudes at $\mathbf{z} \in \Omega$ and $\mathbf{z} \in \mathbb{R}^{2} \backslash \Omega$, respectively.

2.2. Multifrequency MUSIC-Type Imaging Function. We design multifrequency MUSIC-type imaging function and try to describe its structure. First, we introduce a multifrequency MUSIC-type algorithm $\mathbb{E}_{\mathrm{MF}}: \mathbb{C}^{N \times 1} \rightarrow \mathbb{R}$ defined by

$$
\mathbb{E}_{\mathrm{MF}}(\mathbf{z} ; S)=\left(\frac{1}{S} \sum_{s=1}^{S}\left\|\mathbf{P}_{\text {noise }}\left(\mathbf{f}\left(\mathbf{z}, \omega_{s}\right)\right)\right\|^{2}\right)^{-1 / 2} .
$$

Then, we can introduce the following lemma. A more detailed derivation can be found in [16].

Lemma 1 (see [16]). Assume that $k_{S}$ and $S$ are sufficiently large; then,

$$
\mathbb{E}_{M F}(\mathbf{z} ; S) \approx \sqrt{\frac{1}{N}}\left(1-\sum_{m=1}^{M} \Phi\left(\left\|\mathbf{z}-\mathbf{y}_{m}\right\| ; \omega_{1}, \omega_{S}\right)\right)^{-1 / 2},
$$

where function $\Phi\left(x ; \omega_{1}, \omega_{S}\right)$ is defined as

$$
\begin{aligned}
\Phi\left(x ; \omega_{1}, \omega_{S}\right):= & \frac{1}{\omega_{S}-\omega_{1}}\left[\omega_{S}\left(J_{0}\left(\omega_{S} x\right)^{2}+J_{1}\left(\omega_{S} x\right)^{2}\right)\right. \\
& \left.-\omega_{1}\left(J_{0}\left(\omega_{1} x\right)^{2}+J_{1}\left(\omega_{1} x\right)^{2}\right)\right] \\
& +\int_{\omega_{1}}^{\omega_{S}} J_{1}(\omega x)^{2} d \omega .
\end{aligned}
$$

So we can recognize the mathematical structure of multifrequency MUSIC-type algorithm. However, the finite representation of $\int J_{1}^{2}(x) d x$ does not exist. Because of this term, although this can be negligible (see [15]), the map of $\mathbb{E}_{\mathrm{MF}}(\mathbf{z} ; S)$ should generate unexpected points of small magnitudes. In order to solve this problem, the last term of [14] should be 


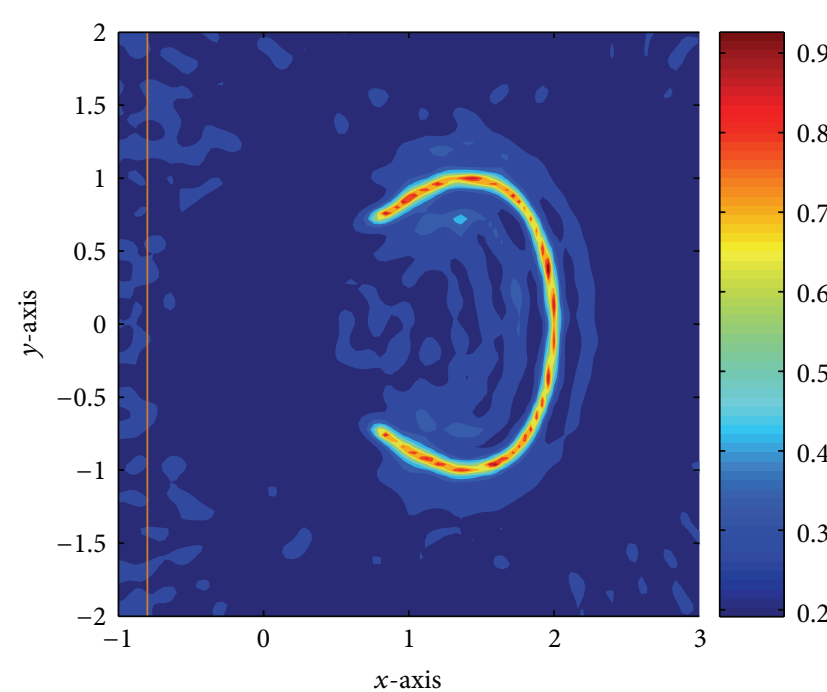

(a) Map of $\mathbb{E}_{\mathrm{MF}}(\mathbf{z} ; 10)$

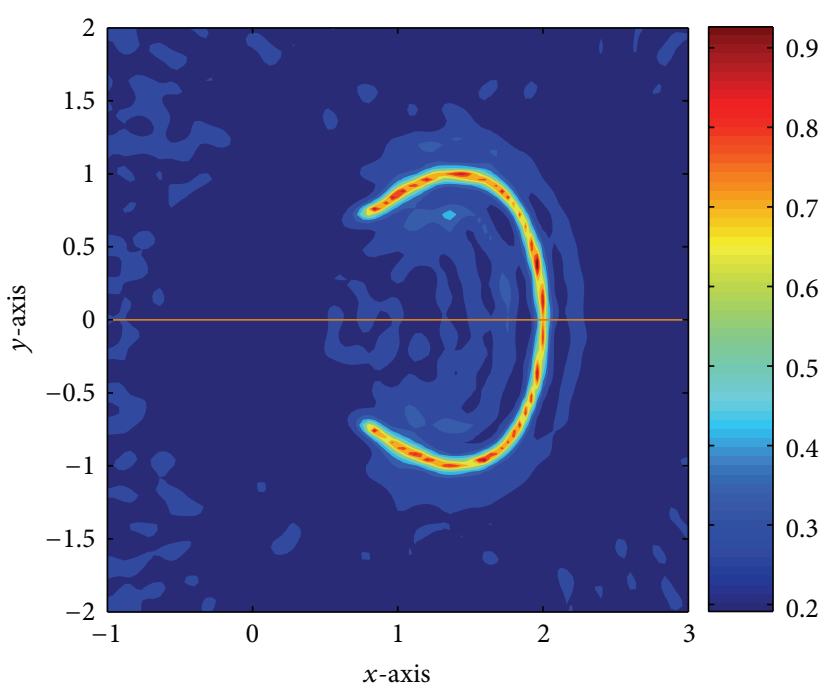

(c) Map of $\mathbb{E}_{\mathrm{MF}}(\mathbf{z} ; 10)$

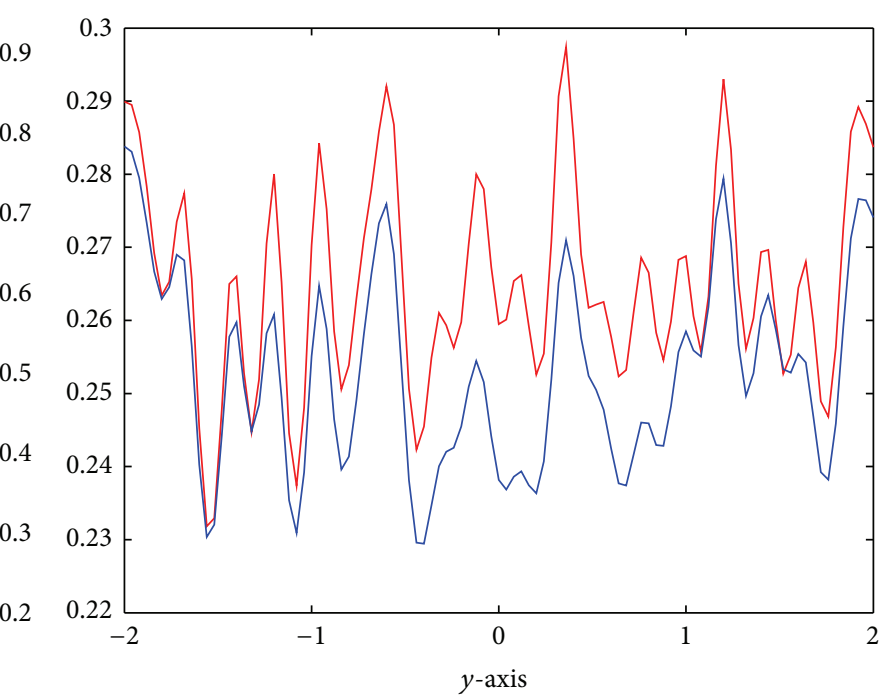

(b) Graph of oscillating pattern

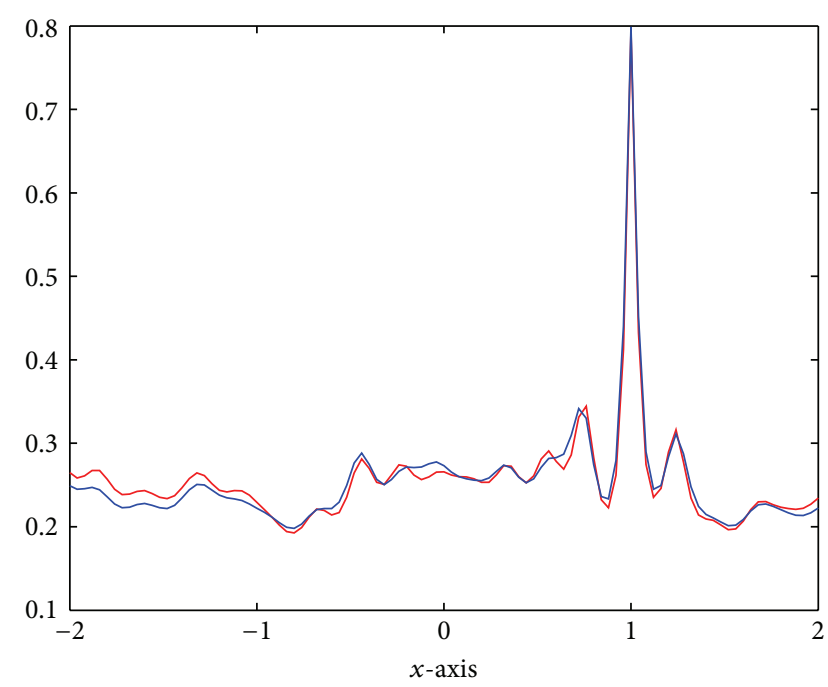

(d) Graph of oscillating pattern

FIGURE 3: Blue- and red-colored lines are $\mathbb{E}_{\mathrm{WMF}}(\mathbf{z} ; 10)$ and $\mathbb{E}_{\mathrm{MF}}(\mathbf{z} ; 10)$, respectively, at $\mathbf{z}=[-0.8, y]^{T}((\mathrm{a})$ and $(\mathrm{b}))$ and $\mathbf{z}=[x, 0]^{T}((\mathrm{c})$ and $(\mathrm{d}))$.

eliminated. For this, we suggest a weighted multifrequency MUSIC-type imaging algorithm in the upcoming section.

\section{Weighted Multifrequency MUSIC-Type Algorithm and Its Structure}

In order to propose the weighted multifrequency MUSICtype imaging algorithm, we introduce the following lemma derived from [16].

Lemma 2 ([16, page 218]). For sufficiently large $N$ and $\omega$, the following relationship holds:

$$
\left\|\mathbf{P}_{\text {noise }}(\mathbf{f}(\mathbf{z}, \omega))\right\| \approx \sqrt{N}\left(1-\sum_{m=1}^{M} J_{0}\left(\omega\left|\mathbf{z}-\mathbf{y}_{m}\right|\right)^{2}\right)^{1 / 2} .
$$

With this, let us define an alternative projection operator weighted by applied frequency $\mathbf{P}_{\mathrm{WN}}: \mathbb{C}^{N \times 1} \rightarrow \mathbb{R}$ as

$$
\mathbf{P}_{\mathrm{WN}}(\mathbf{f}(\mathbf{z}, \omega))=\mathbf{P}_{\text {noise }}(\sqrt{\omega} \mathbf{f}(\mathbf{z}, \omega)) .
$$

Then, the following result can be obtained.

Theorem 3. Assume that $N$ and $\omega$ are sufficiently large; then,

$$
\left\|\mathbf{P}_{W N}(\mathbf{f}(\mathbf{z}, \omega))\right\| \approx \sqrt{N}\left(\omega-\sum_{m=1}^{M} \omega J_{0}\left(\omega\left|\mathbf{z}-\mathbf{y}_{m}\right|\right)^{2}\right)^{1 / 2} .
$$

Proof. The following equations are satisfied by the definition of $\mathbf{P}_{\mathrm{WN}}(\mathbf{f}(\mathbf{z}, \omega))$ and by Lemma 2:

$$
\begin{aligned}
\left\|\mathbf{P}_{\mathrm{WN}}(\mathbf{f}(\mathbf{z}, \omega))\right\| & =\left\|\mathbf{P}_{\text {noise }}(\sqrt{\omega}(\mathbf{f}(\mathbf{z}, \omega)))\right\| \\
& =\sqrt{\omega}\left\|\mathbf{P}_{\text {noise }}((\mathbf{f}(\mathbf{z}, \omega)))\right\|
\end{aligned}
$$




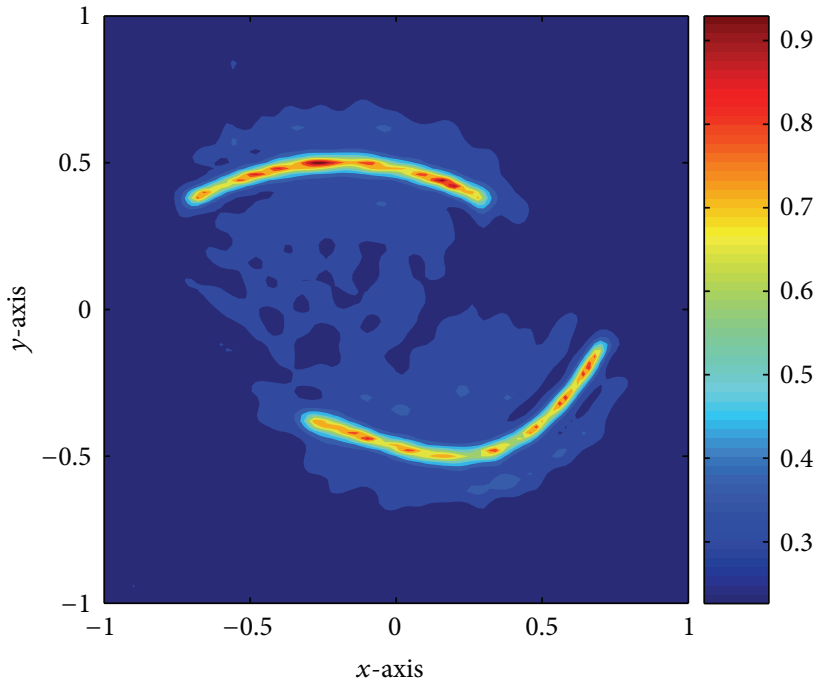

(a) Map of $\mathbb{E}_{\mathrm{MF}}(\mathbf{z} ; 10)$

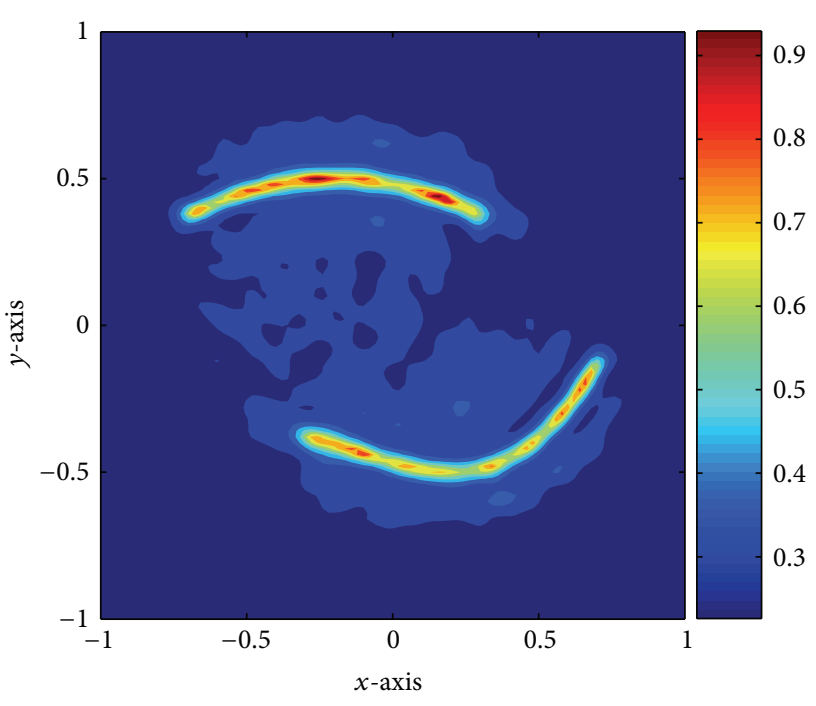

(b) Map of $\mathbb{E}_{\mathrm{WMF}}(\mathbf{z} ; 10)$

FIgURE 4: Same as Figure 1 except the crack is $\Omega_{3}$.

$$
\begin{aligned}
& \approx \sqrt{\omega} \sqrt{N}\left(1-\sum_{m=1}^{M} J_{0}\left(\omega\left|\mathbf{z}-\mathbf{y}_{m}\right|\right)^{2}\right)^{1 / 2} \\
& =\sqrt{N}\left(\omega-\sum_{m=1}^{M} \omega J_{0}\left(\omega\left|\mathbf{z}-\mathbf{y}_{m}\right|\right)^{2}\right)^{1 / 2}
\end{aligned}
$$

Next, we introduce a weighted multifrequency MUSICtype imaging function based on MUSIC-type imaging function $\mathbb{E}_{\mathrm{WMF}}: \mathbb{C}^{N \times 1} \rightarrow \mathbb{R}$ defined by

$$
\mathbb{E}_{W M F}(\mathbf{z} ; S)=\left(\frac{1}{S} \sum_{s=1}^{S}\left\|\mathbf{P}_{\mathrm{WN}}\left(\mathbf{f}\left(\mathbf{z}, \omega_{s}\right)\right)\right\|^{2}\right)^{-1 / 2} .
$$

Then, we can obtain the structure of $\mathbb{E}_{\mathrm{WMF}}(\mathbf{z} ; S)$.

Theorem 4. Assume that $S$ and $\omega_{S}$ are sufficiently large; then,

$$
\begin{aligned}
& \mathbb{E}_{W M F}(\mathbf{z} ; S) \\
& \quad \approx \sqrt{\frac{1}{N}}\left(\frac{\omega_{S}+\omega_{1}}{2}-\sum_{m=1}^{M} \Psi\left(\left|\mathbf{z}-\mathbf{y}_{m}\right| ; \omega_{1}, \omega_{S}\right)\right)^{-1 / 2},
\end{aligned}
$$

where function $\Psi\left(x ; \omega_{1}, \omega_{S}\right)$ is defined as

$$
\begin{aligned}
\Psi\left(x ; \omega_{1}, \omega_{S}\right):=\frac{1}{\omega_{S}-\omega_{1}}\left[\frac{\omega_{S}^{2}}{2}\right. & \left(J_{0}\left(\omega_{S} x\right)^{2}+J_{1}\left(\omega_{S} x\right)^{2}\right) \\
& \left.-\frac{\omega_{1}^{2}}{2}\left(J_{0}\left(\omega_{1} x\right)^{2}+J_{1}\left(\omega_{1} x\right)^{2}\right)\right] .
\end{aligned}
$$

Proof. By Theorem 3, we can calculate the following:

$$
\begin{aligned}
& \mathbb{E}_{\mathrm{WMF}}(\mathbf{z} ; S) \\
& =\left(\frac{1}{S} \sum_{s=1}^{S}\left\|\mathbf{P}_{\mathrm{WN}}\left(\mathbf{f}\left(\mathbf{z}, \omega_{s}\right)\right)\right\|^{2}\right)^{-1 / 2} \\
& \approx\left(\frac{1}{S} \sum_{s=1}^{S}\left(\sqrt{N}\left(\omega-\sum_{m=1}^{M} \omega J_{0}\left(\omega\left|\mathbf{z}-\mathbf{y}_{m}\right|\right)^{2}\right)^{1 / 2}\right)^{2}\right)^{-1 / 2} \\
& =\left(\frac{1}{S} \sum_{s=1}^{S} N\left(\omega-\sum_{m=1}^{M} \omega J_{0}\left(\omega\left|\mathbf{z}-\mathbf{y}_{m}\right|\right)^{2}\right)\right)^{-1 / 2} \\
& =\sqrt{\frac{1}{N}}\left(\sum_{s=1}^{S}\left(\omega-\sum_{m=1}^{M} \omega J_{0}\left(\omega\left|\mathbf{z}-\mathbf{y}_{m}\right|\right)^{2}\right) \frac{1}{S}\right)^{-1 / 2} .
\end{aligned}
$$

Then, since $S$ is sufficiently large, we can observe that

$$
\begin{aligned}
& \sum_{s=1}^{S}\left(\omega-\sum_{m=1}^{M} \omega J_{0}\left(\omega\left|\mathbf{z}-\mathbf{y}_{m}\right|\right)^{2}\right) \frac{1}{S} \\
& \quad \approx \frac{1}{\omega_{S}-\omega_{1}} \int_{\omega_{1}}^{\omega_{S}}\left(\omega-\sum_{m=1}^{M} \omega J_{0}\left(\omega\left|\mathbf{z}-\mathbf{y}_{m}\right|\right)^{2}\right) d \omega,
\end{aligned}
$$

and applying an indefinite integral of the Bessel function (see [24, page 106])

$$
\int x J_{0}(x)^{2} d x=\frac{x^{2}}{2}\left(J_{0}(x)^{2}+J_{1}(x)^{2}\right)
$$




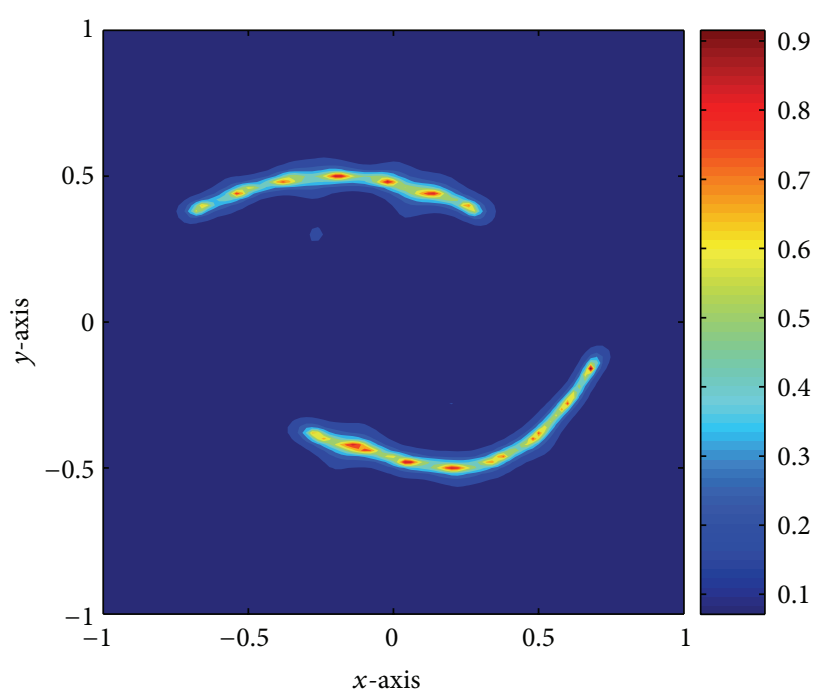

(a) Map of $\mathbb{E}(\mathbf{z})$

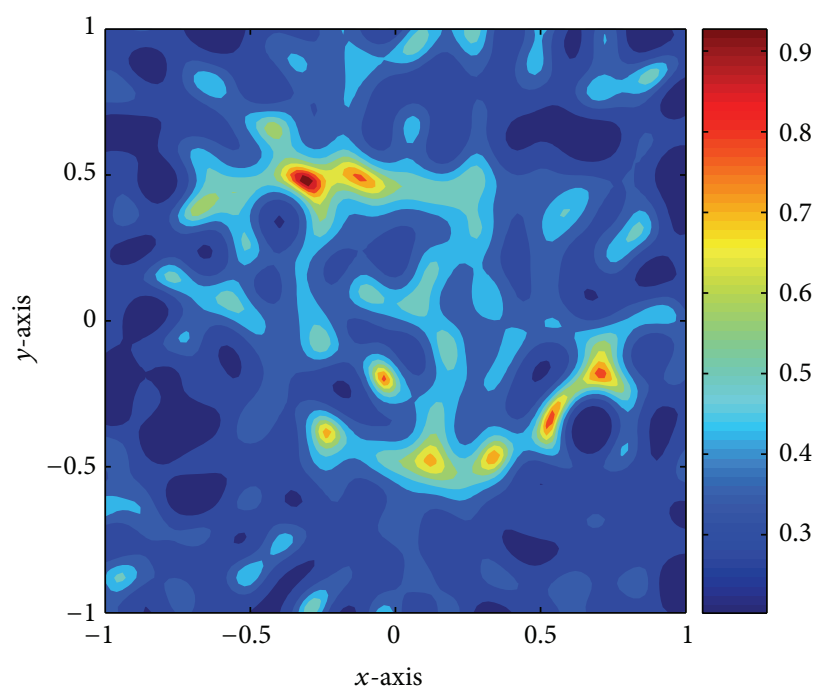

(c) Map of $\mathbb{E}(\mathbf{z})$

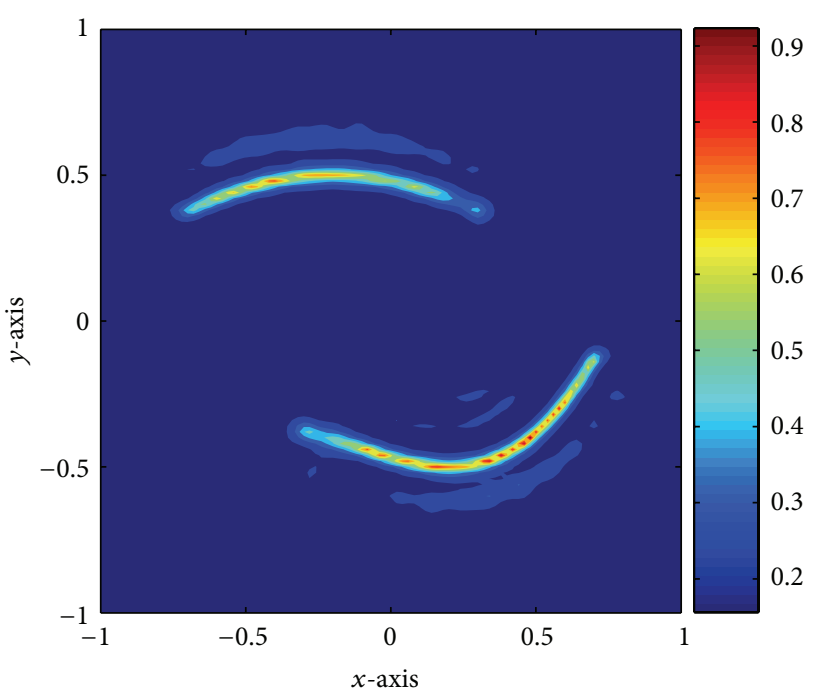

(b) Map of $\mathbb{E}_{\mathrm{WMF}}(\mathbf{z} ; 10)$

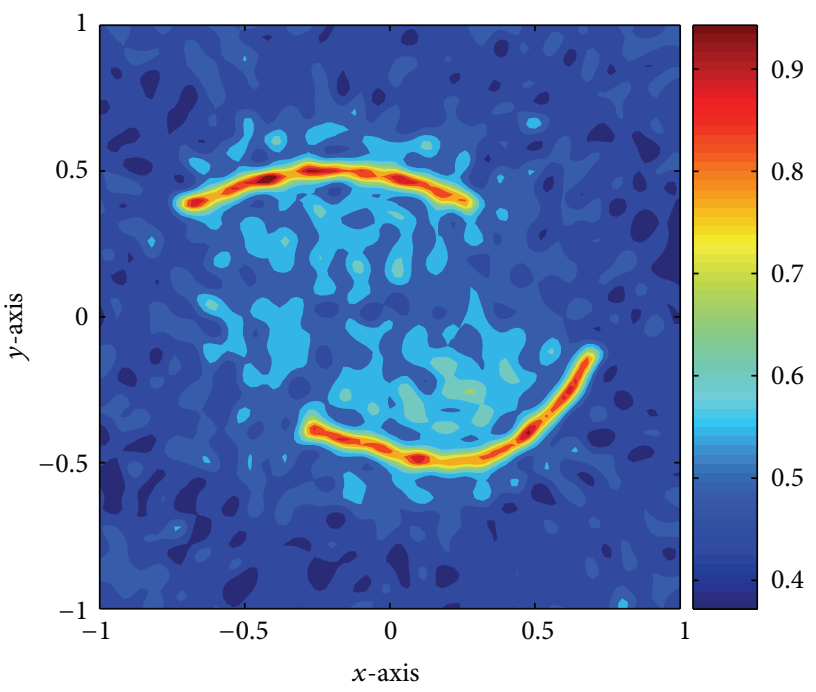

(d) Map of $\mathbb{E}_{\mathrm{WMF}}(\mathbf{z} ; 10)$

FIGURE 5: Influence of noise. $30 \mathrm{~dB}((\mathrm{a})$ and (b)) and $10 \mathrm{~dB}((\mathrm{c})$ and (d)) white Gaussian random is added.

yields

$$
\begin{aligned}
& \frac{1}{\omega_{S}-\omega_{1}} \int_{\omega_{1}}^{\omega_{S}} \omega J_{0}\left(\omega\left|\mathbf{z}-\mathbf{y}_{m}\right|\right)^{2} d \omega \\
& =\frac{1}{\omega_{S}-\omega_{1}}\left[\frac{\omega_{S}^{2}}{2}\left(J_{0}\left(\omega_{S}\left|\mathbf{z}-\mathbf{y}_{m}\right|\right)^{2}+J_{1}\left(\omega_{S}\left|\mathbf{z}-\mathbf{y}_{m}\right|\right)^{2}\right)\right. \\
& \quad-\frac{\omega_{1}^{2}}{2}\left(J_{0}\left(\omega_{1}\left|\mathbf{z}-\mathbf{y}_{m}\right|\right)^{2}\right. \\
& \left.\left.\quad+J_{1}\left(\omega_{1}\left|\mathbf{z}-\mathbf{y}_{m}\right|\right)^{2}\right)\right] \\
& =\Psi\left(\omega\left|\mathbf{z}-\mathbf{y}_{m}\right| ; \omega_{1}, \omega_{S}\right) .
\end{aligned}
$$

Hence, we can obtain

$$
\begin{gathered}
\frac{1}{\omega_{S}-\omega_{1}} \int_{\omega_{1}}^{\omega_{S}}\left(\omega-\sum_{m=1}^{M} \omega J_{0}\left(\omega\left|\mathbf{z}-\mathbf{y}_{m}\right|\right)^{2}\right) d \omega \\
=\frac{\omega_{S}+\omega_{1}}{2}-\sum_{m=1}^{M} \Psi\left(\omega\left|\mathbf{z}-\mathbf{y}_{m}\right| ; \omega_{1}, \omega_{S}\right) .
\end{gathered}
$$

Therefore,

$$
\mathbb{E}_{\mathrm{WMF}}(\mathbf{z} ; S) \approx \sqrt{\frac{1}{N}}\left(\frac{\omega_{S}+\omega_{1}}{2}-\sum_{m=1}^{M} \Psi\left(\left|\mathbf{z}-\mathbf{y}_{m}\right| ; \omega_{1}, \omega_{S}\right)\right)^{-1 / 2} .
$$

This completes the proof.

Looking at the results of Theorem 4, in contrast to the $\mathbb{E}_{\mathrm{MF}}(\mathbf{z} ; S)$, the $\mathbb{E}_{\mathrm{WMF}}(\mathbf{z} ; S)$ does not have the $\int J_{1}^{2}(x) d x$ 


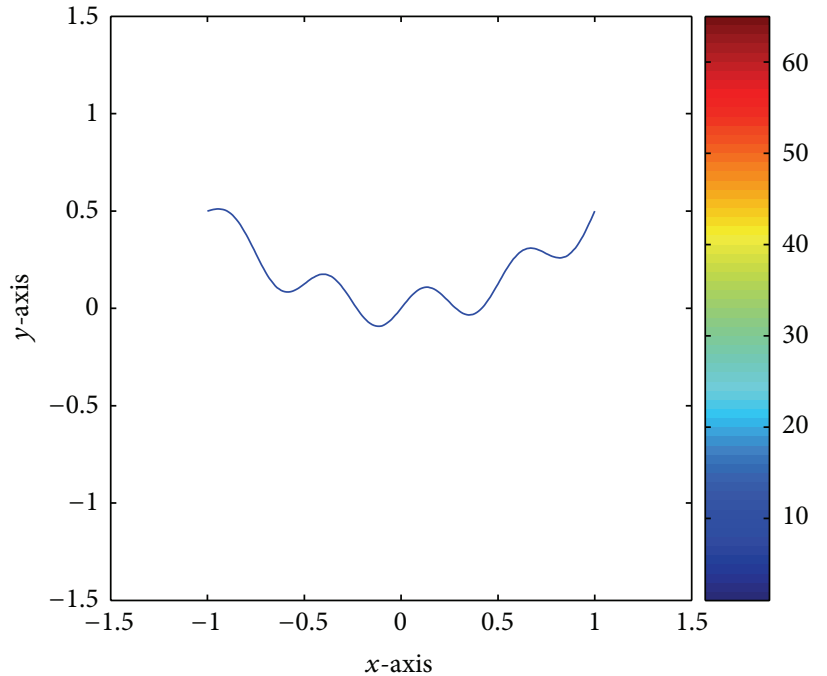

(a) Crack $\Omega_{4}$

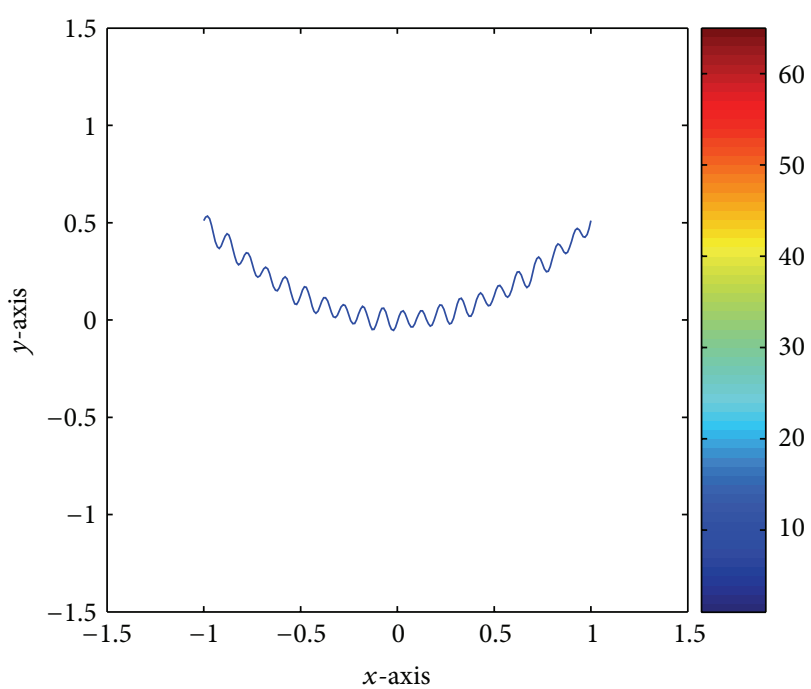

(b) $\operatorname{Crack} \Omega_{5}$

FIGURE 6: Shape of oscillating cracks $\Omega_{4}$ and $\Omega_{5}$.

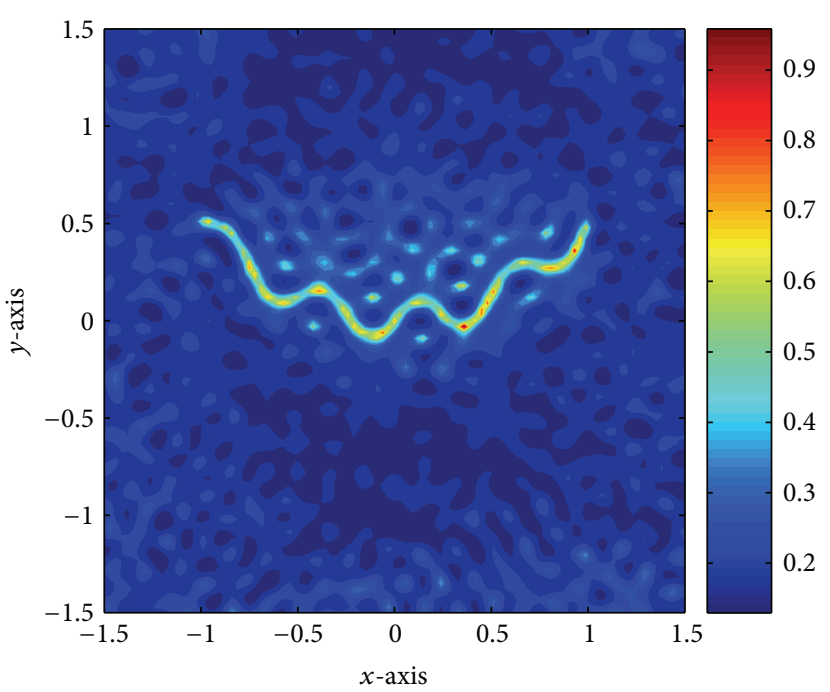

(a) Map of $\mathbb{E}(\mathbf{z})$

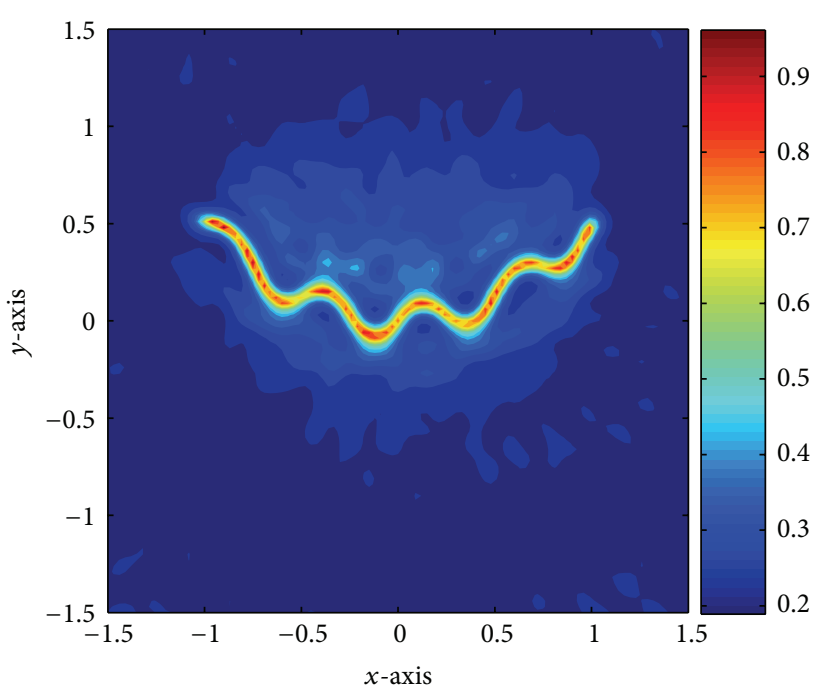

(b) Map of $\mathbb{E}_{\mathrm{WMF}}(\mathbf{z} ; 10)$

FIGURE 7: Shape reconstruction of $\Omega_{4}$ via $\mathbb{E}(\mathbf{z})(a)$ and $\mathbb{E}_{\mathrm{WMF}}(\mathbf{z} ; 10)$ (b).

term. Therefore, we expect that the imaging results of the $\mathbb{E}_{\mathrm{WMF}}(\mathbf{z} ; S)$ will be better than $\mathbb{E}_{\mathrm{MF}}(\mathbf{z} ; S)$. In the next section, numerical experiments will be presented to support this.

\section{Numerical Experiments}

In this section, some numerical examples are displayed in order to support our analysis in the previous section. Applied frequencies are of the form $\omega_{s}=2 \pi / \lambda_{s}$, where $\lambda_{s}, s=$ $1,2, \ldots, S(=10)$ is the given wavelength. The observation directions $\boldsymbol{\theta}_{n} \in \mathbb{S}^{1}$ are taken as

$$
\boldsymbol{\theta}_{n}=\left[\cos \frac{2 \pi n}{N}, \sin \frac{2 \pi n}{N}\right]^{T} .
$$

For illustrating arc-like cracks, three $\Omega_{l}$ are chosen:

$$
\begin{aligned}
& \Omega_{1}=\left\{\left[s, \frac{1}{2} \cos \frac{s \pi}{2}+\frac{1}{5} \sin \frac{s \pi}{2}-\frac{1}{10} \cos \frac{3 s \pi}{2}\right]^{T}:\right. \\
& s \in[-1,1]\}, \\
& \Omega_{2}=\left\{\left[2 \sin \frac{s}{2}, \sin s\right]^{T}: s \in\left[\frac{\pi}{4}, \frac{7 \pi}{4}\right]\right\}, \\
& \Omega_{3}=\Omega_{3}^{(1)} \cup \Omega_{3}^{(2)},
\end{aligned}
$$




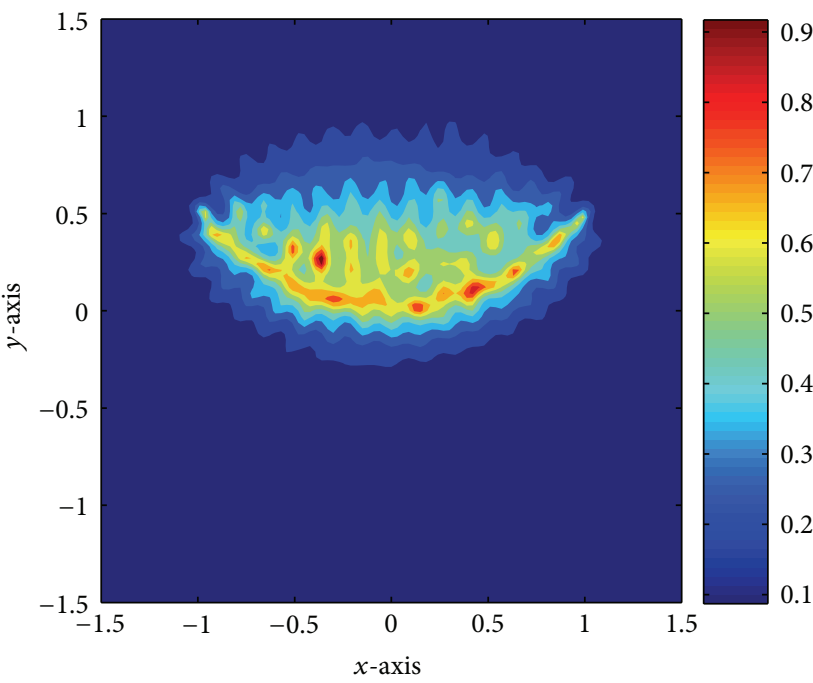

(a) Map of $\mathbb{E}(\mathbf{z})$

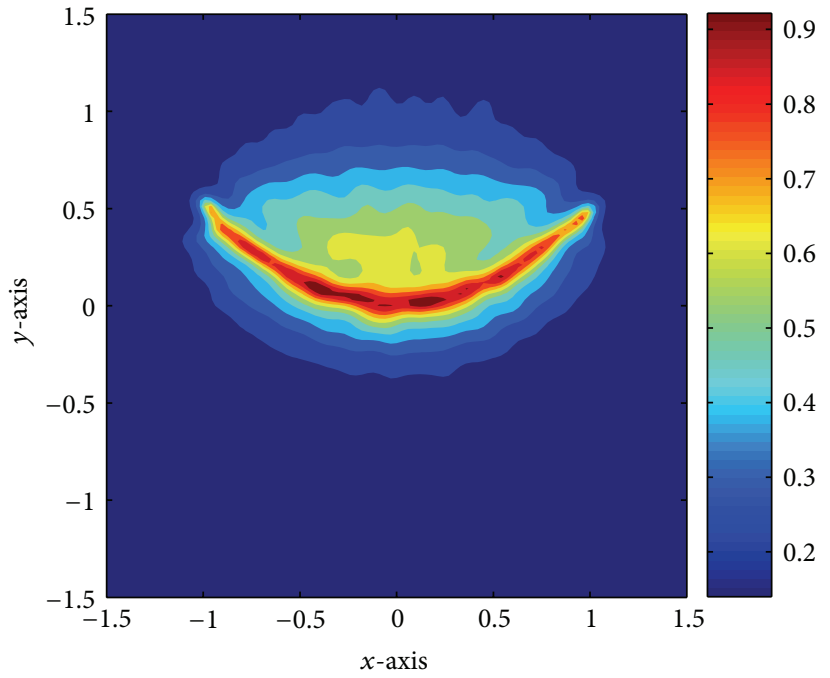

(b) Map of $\mathbb{E}_{\mathrm{WMF}}(\mathbf{z} ; 10)$

FIgURE 8: Same as Figure 7 except the crack is $\Omega_{5}$.

where

$$
\begin{aligned}
& \Omega_{3}^{(1)}=\left\{\left[s-\frac{1}{5},-\frac{s^{2}}{2}+\frac{3}{5}\right]^{T}: s \in\left[-\frac{1}{2}, \frac{1}{2}\right]\right\}, \\
& \Omega_{3}^{(2)}=\left\{\left[s+\frac{1}{5}, s^{3}+s^{2}-\frac{3}{5}\right]^{T}: s \in\left[-\frac{1}{2}, \frac{1}{2}\right]\right\} .
\end{aligned}
$$

It is worth emphasizing that all the far-field data $u_{\text {far }}$ of (6) are generated by the method introduced in [25, Chapter 3, 4]. After generating the data, a $20 \mathrm{~dB}$ white Gaussian random noise is added to the unperturbed data. In order to obtain the number of nonzero singular values $M$ for each frequency, a 0.1 -threshold scheme (choosing first $m$ singular values $\tau_{m}$ such that $\tau_{m} / \tau_{1} \geq 0.1$ ) is adopted. A more detailed discussion of thresholding can be found in [20,22].

Figures 1 and 2 show the imaging results via multifrequency MUSIC and weighted multifrequency MUSIC algorithms for single crack $\Omega_{1}$ and $\Omega_{2}$, respectively. As we already mentioned, since the term $\int J_{1}^{2}(x) d x$ can be disregarded, it is very hard to compare the improvements via visual inspection of the reconstructions. However, based on Figure 3, we can examine that the proposed weighted multifrequency MUSIC algorithm successfully reduces these artifacts, so we can conclude that this is an improved version.

Figure 4 shows the imaging results via multifrequency MUSIC and weighted multifrequency MUSIC algorithms for multiple cracks $\Omega_{3}$. Similar to the imaging of single crack, we can observe that weighted multifrequency MUSIC algorithm improves the traditional one, although it is hard to compare the improvements via visual inspection.

Figure 5 shows the noise contribution in terms of SNR. In order to observe the effect of noise, $30 \mathrm{~dB}$ and $10 \mathrm{~dB}$ white Gaussian random noises are added to the unperturbed data. Based on these results, we can easily observe that both traditional and proposed MUSIC algorithms offer very good result when $30 \mathrm{~dB}$ noise is added. However, when $10 \mathrm{~dB}$ noise is added, the traditional MUSIC algorithm yields a poor result while the proposed algorithm yields an acceptable result.

Now, we consider the imaging of oscillating crack. For this, we consider the following cracks (see Figure 6):

$$
\begin{gathered}
\Omega_{4}=\left\{\left[s, \frac{1}{2} s^{2}+\frac{1}{10} \sin (4 \pi(s+1))\right]^{T}: s \in[-1,1]\right\} \\
\Omega_{5}=\left\{\left[s, \frac{1}{2} s^{2}+\frac{1}{20} \sin (20 \pi(s+1))-\frac{1}{100} \cos (15 \pi s)\right]^{T}:\right. \\
s \in[-1,1]\} .
\end{gathered}
$$

Figure 7 shows the maps of $\mathbb{E}(\mathbf{z})$ and $\mathbb{E}_{\mathrm{WMF}}(\mathbf{z} ; 10)$ for the crack $\Omega_{4}$. In this result, we can observe that both traditional and proposed MUSIC algorithms produce acceptable result, but the proposed algorithm successfully eliminates replicas.

Figure 8 shows the maps of $\mathbb{E}(\mathbf{z})$ and $\mathbb{E}_{\mathrm{WMF}}(\mathbf{z} ; 10)$ for highly oscillating crack $\Omega_{5}$. Opposite to Figure 7, both traditional and proposed MUSIC algorithms yield poor result. This example shows the limitation of proposed algorithm.

\section{Conclusion}

Based on the structure of multifrequency MUSIC-type imaging function, we introduced a weighted multifrequency MUSIC-type imaging function. Through a careful analysis, a relationship between imaging function and Bessel function of the first kind of integer order is established, and we have confirmed that the proposed imaging algorithm is an improved version of the traditional one.

Although, the proposed algorithm produces very good results and improves the traditional MUSIC algorithm, it still needs some upgrade, for example, imaging of highly oscillating cracks. Development of this should be an interesting 
and remarkable research project. In this paper, we considered the MUSIC algorithm in full-view inverse scattering problem. Based on the result in [26], the MUSIC algorithm cannot be applied to limited-view problems but the reason is still unknown. Identifying the structure of the MUSIC algorithm in the limited-view inverse scattering problems will be the forthcoming work.

\section{Acknowledgments}

The author would like to express his thanks to Won-Kwang Park (Kookmin University) for his valuable discussions and MATLAB simulations to generate the forward data and MUSIC-type imaging function. The author would like to acknowledge two anonymous referees for their precious comments. This work was supported by the Basic Science Research Program through the National Research Foundation of Korea (NRF) funded by the Ministry of Education, Science and Technology (no. 2011-0007705) and the research program of Kookmin University in Korea.

\section{References}

[1] I. Arnedo, I. Arregui, M. Chudzik et al., "Passive microwave component design using inverse scattering: theory and applications," International Journal of Antennas and Propagation, vol. 2013, Article ID 761278, 10 pages, 2013.

[2] H. Ammari, E. Iakovleva, and D. Lesselier, "A music algorithm for locating small inclusions buried in a half-space from the scattering amplitude at a fixed frequency," Multiscale Modeling and Simulation, vol. 3, no. 3, pp. 597-628, 2005.

[3] H. Ammari, H. Kang, E. Kim, K. Louati, and M. S. Vogelius, "A MUSIC-type algorithm for detecting internal corrosion from electrostatic boundary measurements," Numerische Mathematik, vol. 108, no. 4, pp. 501-528, 2008.

[4] O. Dorn and D. Lesselier, "Level set methods for inverse scattering," Inverse Problems, vol. 22, no. 4, article R01, pp. R67R131, 2006.

[5] J. M. Geffrin, P. C. Chaumet, C. Eyraud, K. Belkebir, and P. Sabouroux, "Electromagnetic three-dimensional reconstruction of targets from free space experimental data," Applied Physics Letters, vol. 92, no. 19, Article ID 194103, 2008.

[6] S. Hou, K. Huang, K. Sølna, and H. Zhao, "A phase and space coherent direct imaging method," Journal of the Acoustical Society of America, vol. 125, no. 1, Article ID 125206, pp. 227238, 2009.

[7] V. A. Markel and J. C. Schotland, "Effects of sampling and limited data in optical tomography," Applied Physics Letters, vol. 81, no. 7, pp. 1180-1182, 2002.

[8] B. Scholz, "Towards virtual electrical breast biopsy: spacefrequency MUSIC for trans-admittance data," IEEE Transactions on Medical Imaging, vol. 21, no. 6, pp. 588-595, 2002.

[9] G. Ventura, J. X. Xu, and T. Belytschko, "A vector level set method and new discontinuity approximations for crack growth by EFG," International Journal for Numerical Methods in Engineering, vol. 54, no. 6, pp. 923-944, 2002.

[10] R. Kress, "Inverse scattering from an open arc," Mathematical Methods in the Applied Sciences, vol. 18, no. 4, pp. 267-293, 1995.
[11] C. T. Kelley, Iterative Methods for Optimization, vol. 18, Society for Industrial and Applied Mathematics, Philadelphia, Pa, USA, 1999.

[12] T. Henriksson, M. Lambert, and D. Lesselier, "MUSICtype algorithm for eddy-current nondestructive evaluation of small defects in metal plates," in Dans Electromagnetic NonDestructive Evaluation, vol. 25 of Studies in Applied Electromagnetics and Mechanics, pp. 22-29, 2011.

[13] S. Hou, K. Solna, and H. Zhao, "A direct imaging algorithm for extended targets," Inverse Problems, vol. 22, no. 4, pp. 1151-1178, 2006.

[14] M. Cheney, "The linear sampling method and the MUSIC algorithm," Inverse Problems, vol. 17, no. 4, pp. 591-595, 2001.

[15] Y. D. Joh, Y. M. Kwon, J. Y. Huh, and W. K. Park, "Structure analysis of single- and multi-frequency subspace migrations in inverse scattering problems," Progress in Electromagnetics Research, vol. 136, pp. 607-622, 2013.

[16] Y. D. Joh and W. K. Park, "Structural behavior of the music-type algorithm for imaging perfectly conducting cracks," Progress in Electromagnetics Research, vol. 138, pp. 211-226, 2013.

[17] Y. K. Ma, P. S. Kim, and W. K. Park, "Analysis of topological derivative function for a fast electromagnetic imaging of perfectly conducing cracks," Progress in Electromagnetics Research, vol. 122, pp. 311-325, 2012.

[18] Y. K. Ma and W. K. Park, "A topological derivative based non-iterative electromagnetic imaging of perfectly conducting cracks," Journal of Electromagnetic Engineering and Science, vol. 12, no. 1, pp. 128-134, 2012.

[19] R. Solimene, G. Ruvio, A. Dell' Aversano, A. Cuccaro, M. J. Ammann, and R. Pierri, "Detecting point-like sources of unknown frequency spectra," Progress in Electromagnetics Research B, vol. 50, pp. 347-364, 2013.

[20] W. K. Park and D. Lesselier, "Electromagnetic MUSIC-type imaging of perfectly conducting, arc-like cracks at single frequency," Journal of Computational Physics, vol. 228, no. 21, pp. 8093-8111, 2009.

[21] E. Beretta and E. Francini, "Asymptotic formulas for perturbations in the electromagnetic fields due to the presence of thin inhomogeneities," Contemporary Mathematics, vol. 333, pp. 4963, 2003.

[22] W. K. Park and D. Lesselier, "Reconstruction of thin electromagnetic inclusions by a level-set method," Inverse Problems, vol. 25, no. 8, Article ID 085010, 2009.

[23] H. Ammari, H. Kang, H. Lee, and W. K. Park, "Asymptotic imaging of perfectly conducting cracks," SIAM Journal on Scientific Computing, vol. 32, no. 2, pp. 894-922, 2010.

[24] W. Rosenheinrich, "Tables of some indefinite integrals of bessel functions," http://www.fh-jena.de/ rsh/Forschung/Stoer/besint.pdf.

[25] Z. T. Nazarchuk, Singular Integral Equations in Diffraction Theory, Karpenko Physicomechanical Institute, Ukrainian Academy of Sciences, Lviv, Ukraine, 1994.

[26] W. K. Park, "On the imaging of thin dielectric inclusions buried within a half-space," Inverse Problems, vol. 26, no. 7, Article ID 074008, 2010. 


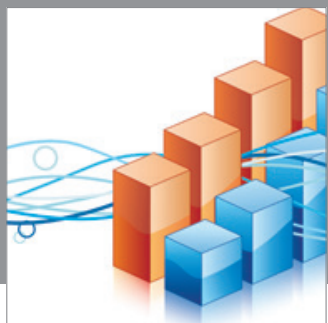

Advances in

Operations Research

mansans

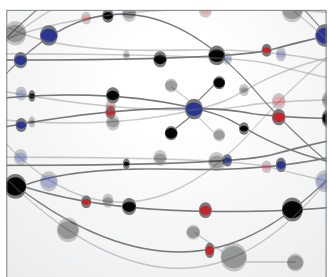

The Scientific World Journal
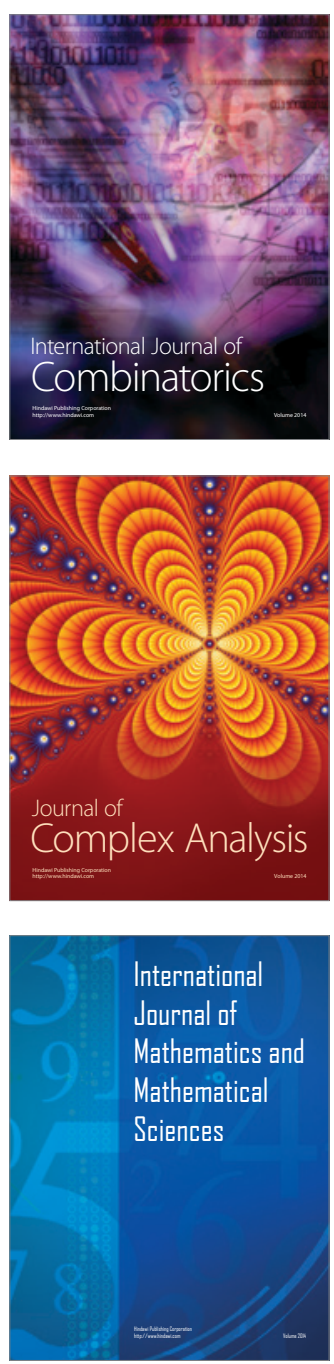
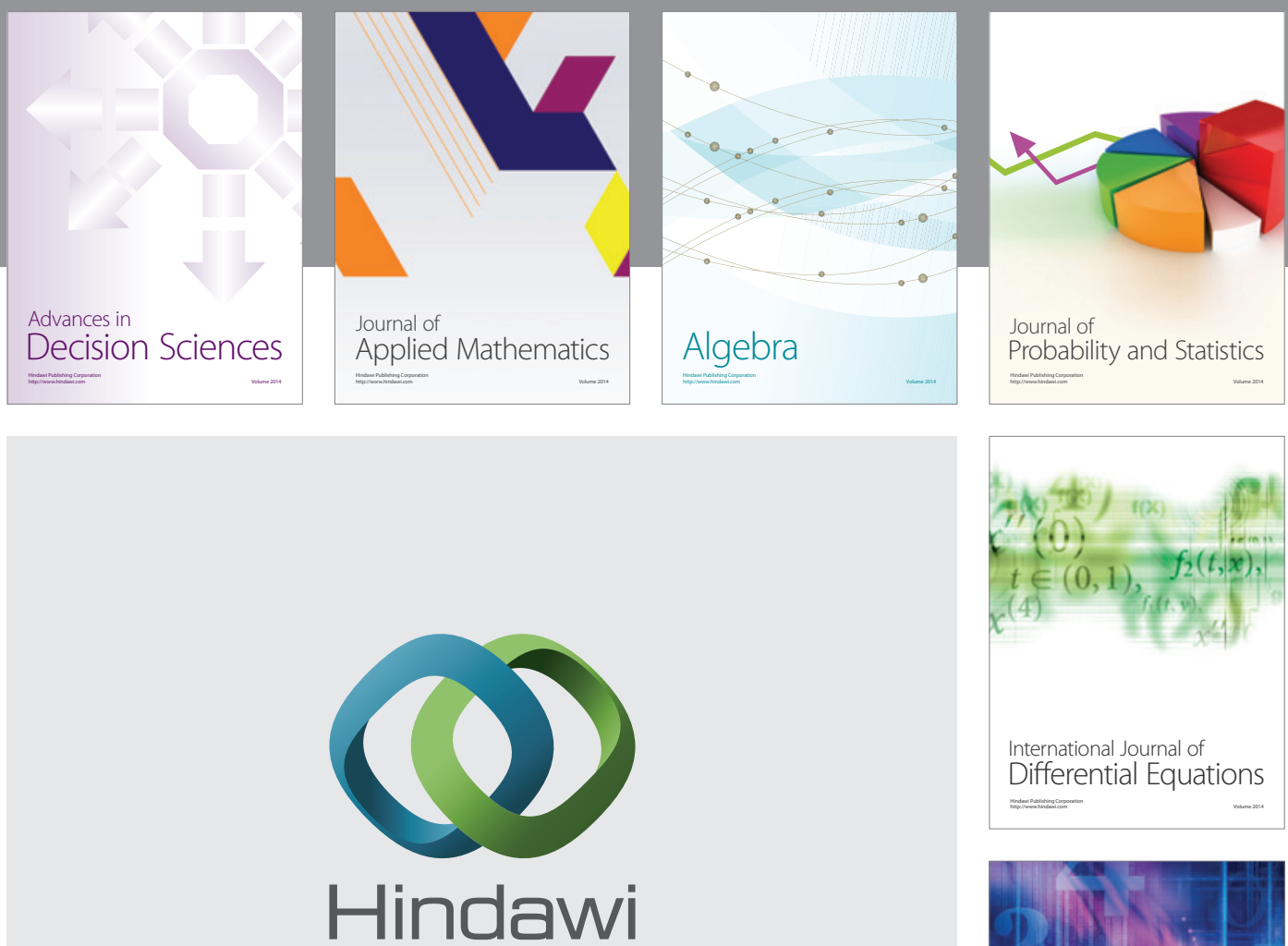

Submit your manuscripts at http://www.hindawi.com
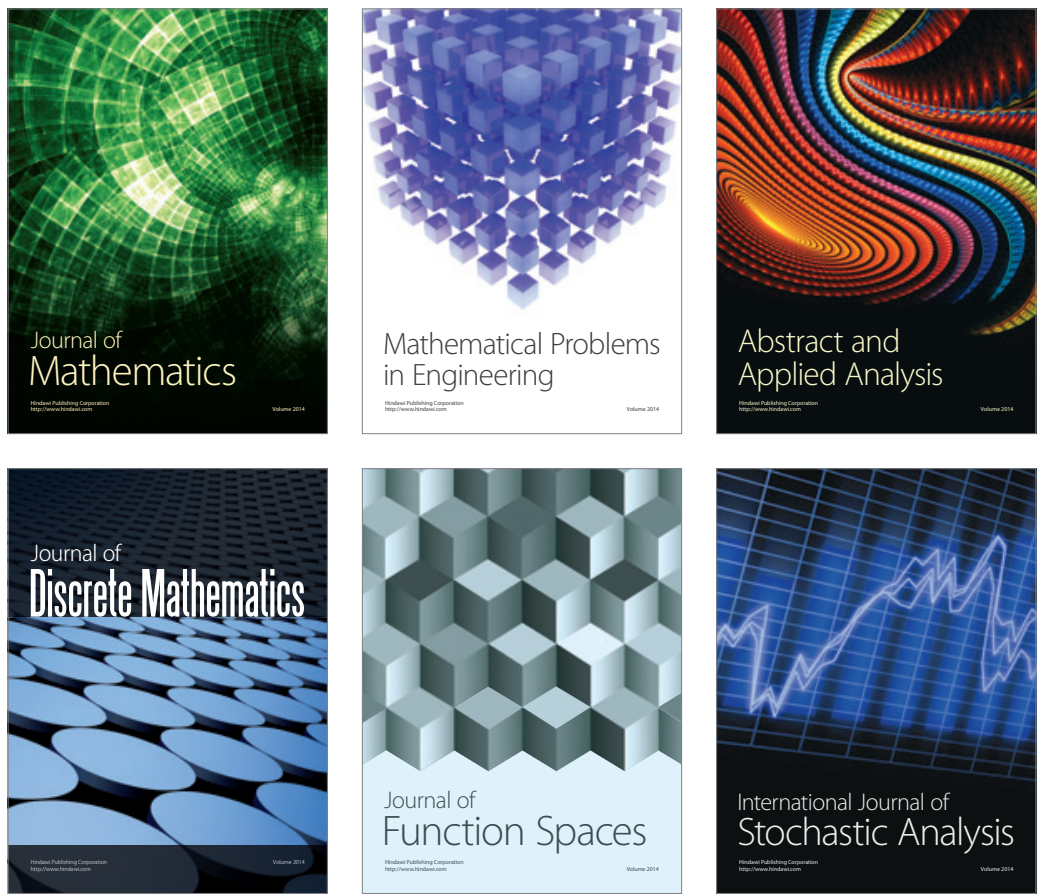

Journal of

Function Spaces

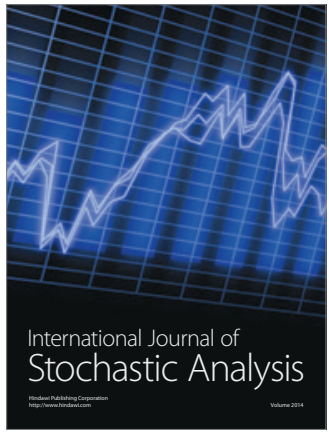

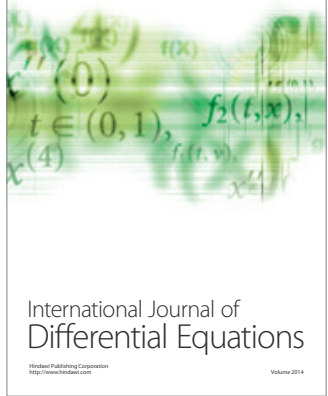
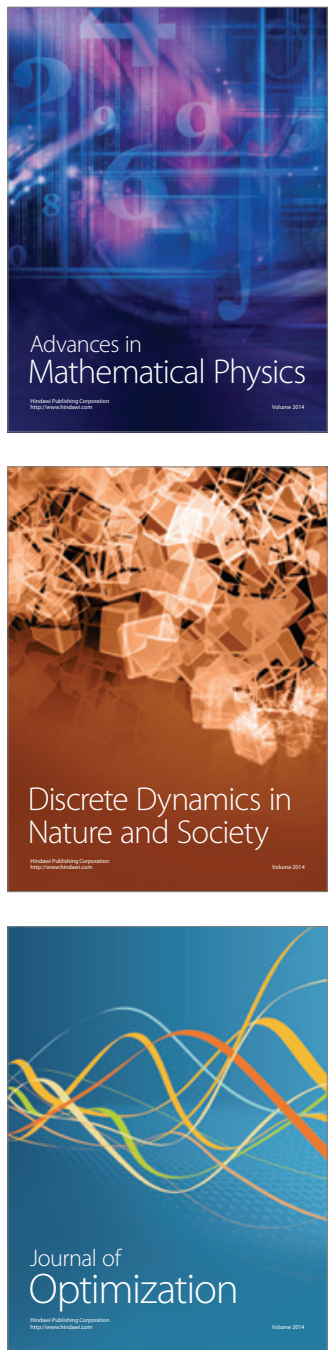